the susceptible ICR strain but not in adult mice or the resistant C57BL/6 strain (8). Similarly, in humans, the loss of one kidney has minimal consequences in healthy adults, whereas children with nephrectomy at a young age have markedly increased incidence of albuminuria and hypertension at follow-up $(16,17)$. The incidence of CKD, particularly hypertension-associated or due to FSGS, is markedly increased in African Americans compared with those of European descent (17). Various factors have been proposed, including an APOL1 gene variant and low birth weight linked to low nephron number $(17,18)$. Whether mTORC1 alterations could influence human nephron development and also later susceptibility to additional injuries is not known. Additional signaling pathways such as the Notch, PPAR $\gamma$, and calcineurin pathways also modulate the podocyte and its injury responses (19-21). Interactions of these pathways with mTORC actions in podocytes are not yet known.

\section{Clinical implications moving forward}

The challenge is to identify specific disease stages for beneficial manipulation of mTOR pathway activity. Taken together, the work of Gödel et al. (8) and Inoki et al. (9) demonstrate that too little or too much mTOR activity is deleterious to the podocyte, analogous to the fine-tuned balance of VEGF actions on the glomerulus. The findings add yet another complex dimension to the understanding of the dynamic regula- tion of the podocyte. Further in-depth examination of mechanisms in experimental models that faithfully capture key elements of human disease counterparts, and ultimately translation to human conditions, will be necessary.

\section{Acknowledgments}

This work was supported in part by NIH grants NIDDK R01DK56942 and P50 DK44757.

Address correspondence to: Agnes B. Fogo, MCN C3310, Department of Pathology, Vanderbilt University Medical Center, Nashville, Tennessee 37232, USA. Phone: 615.322.3070; Fax: 615.343.7023; E-mail: agnes.fogo@vanderbilt.edu.

1. Mathieson PW. Update on the podocyte. Curr Opin Nephrol Hypertens. 2009;18(3):206-211.

2. Ohse $\mathrm{T}$, et al. The enigmatic parietal epithelial cell is finally getting noticed: a review. Kidney Int. 2009;76(12):1225-1238.

3. Fatima H, Moeller MJ, Smeets B, Yang H-C, Fogo AB. Parietal epithelial cell activation distinguishes early recurrence of FSGS in the transplant from minimal change disease. (abstract 1460) Lab Invest. 2011;91(1):343A.

4. Smeets B, Dijkman HB, Wetzels JF, Steenbergen EJ. Lessons from studies on focal segmental glomerulosclerosis: an important role for parietal epithelial cells? J Pathol. 2006;210(3):263-272.

5. Fioretto P, Steffes MW, Sutherland DE, Goetz FC, Mauer M. Reversal of lesions of diabetic nephropathy after pancreas transplantation. N Engl J Med. 1998; 339(2):69-75

6. Alpers CE, Hudkins KL. Mouse models of diabetic nephropathy. Curr Opin Nephrol Hypertens. 2011; 20(3):278-284.

7. Fogo AB. Can glomerulosclerosis be reversed? Nat
Clin Pract Nephrol. 2006;2(6):290-291.

8. Gödel M, et al. Role of mTOR in podocyte function and diabetic nephropathy in humans and mice. J Clin Invest. 2011;121(6):2197-2209.

9. Inoki $\mathrm{K}$, et al. mTORC1 activation in podocytes is a critical step in the development of diabetic nephropathy in mice. J Clin Invest. 2011;121(6):2181-2196.

10. Huber TB, Walz G, Kuehn EW. mTOR and rapamycin in the kidney: signaling and therapeutic implications beyond immunosuppression. Kidney Int. 2011;79(5):502-511.

11. Yamaguchi Y, et al. Epithelial-mesenchymal transition as a potential explanation for podocyte depletion in diabetic nephropathy. Am J Kidney Dis. 2009;54(4):653-664.

12. Yang $H$, Fogo AB. Cell senescence in the aging kidney. J Am Soc Nephrol. 2010;21(9):1436-1439.

13. Ikoma M, Kawamura T, Kakinuma Y, Fogo A, Ichikawa I. Cause of variable therapeutic efficiency of angiotensin converting enzyme inhibitor on glomerular lesions. Kidney Int. 1991;40(2):195-202.

14. Matsusaka T, Kobayashi K, Kon V, Pastan I, Fogo AB, Ichikawa I. Glomerular sclerosis is prevented during urinary tract obstruction due to podocyte protection. Am J Physiol Renal Physiol. 2011;300(3):F792-F800.

15. Matsusaka T, et al. Study of genetically engineered chimeric glomeruli revealed podocyte damage damages other podocytes. J Am Soc Nephrol. In press.

16. Di Tullio MT, et al. Compensatory hypertrophy and progressive renal damage in children nephrectomized for Wilms' tumor. Med Pediatr Oncol. 1996; 26(5):325-328.

17. Marcantoni C, Fogo AB. A perspective on arterionephrosclerosis: from pathology to potential pathogenesis. J Nephrol. 2007;20(5):518-524.

18. Genovese $G$, et al. Association of trypanolytic ApoL1 variants with kidney disease in African Americans. Science. 2010;329(5993):841-845.

19. Murea M, et al. Expression of Notch pathway proteins correlates with albuminuria, glomerulosclerosis, and renal function. Kidney Int. 2010;78(5):514-522.

20. Kanjanabuch T, et al. PPAR-gamma agonist protects podocytes from injury. Kidney Int. 2007; 71(12):1232-1239.

21. Faul C, et al. The actin cytoskeleton of kidney podocytes is a direct target of the antiproteinuric effect of cyclosporine A. Nat Med. 2008;14(9):931-938.

\title{
Spotlight on childhood blindness
}

\author{
José-Alain Sahel1,2,3,4,5,6
}

${ }^{1}$ INSERM, U968, Paris, France. ${ }^{2}$ Université Pierre et Marie Curie-Paris6, UM80, Institut de la Vision, Paris, France. ${ }^{3}$ CNRS, UMR 7210, Paris, France. ${ }^{4}$ Centre Hospitalier National d'Ophtalmologie des Quinze-Vingts, INSERM-DHOS CIC 503, Paris, France. ${ }^{5}$ Fondation Ophtalmologique Adolphe de Rothschild, Paris, France. ${ }^{6}$ Institute of Ophthalmology, University College of London, London, United Kingdom.

\begin{abstract}
Leber congenital amaurosis (LCA) is a rare disease that severely affects vision in early life. It is characterized by genetic and clinical heterogeneity due to complex and not fully understood pathogenetic mechanisms. It is also now widely known as a disease model for gene therapy. In this issue of the JCI, two independent research groups report valuable new data on LCA. Specifically, they provide important insights into the pathophysiological mechanisms of LCA and offer strong hope that the outcome of gene therapy for retinal degenerative diseases will be successful.
\end{abstract}

Conflict of interest: The author receives research support and income from Fovea-Pharmaceuticals-Sanofi.

Citation for this article: J Clin Invest. 2011;

121(6):2145-2149. doi:10.1172/JCI58300.

\section{Highlights of LCA}

Leber congenital amaurosis (LCA) is a rare retinal dystrophy with a prevalence at birth of approximately 1 in 80,000 (1). It is most often inherited in an autosomal-recessive manner. It typically becomes evident in the first year of life, with poor visual function often accompanied by nystagmus, sluggish or near-absent pupillary responses, photophobia, high hyperopia, and keratoconus. Profound visual impairment is usually present from birth.

LCA is caused by mutations in any one of at least 15 genes (2), and this accounts in part for its heterogeneous clinical presentation. To date, more than 400 mutations have been identified. Together, they account 


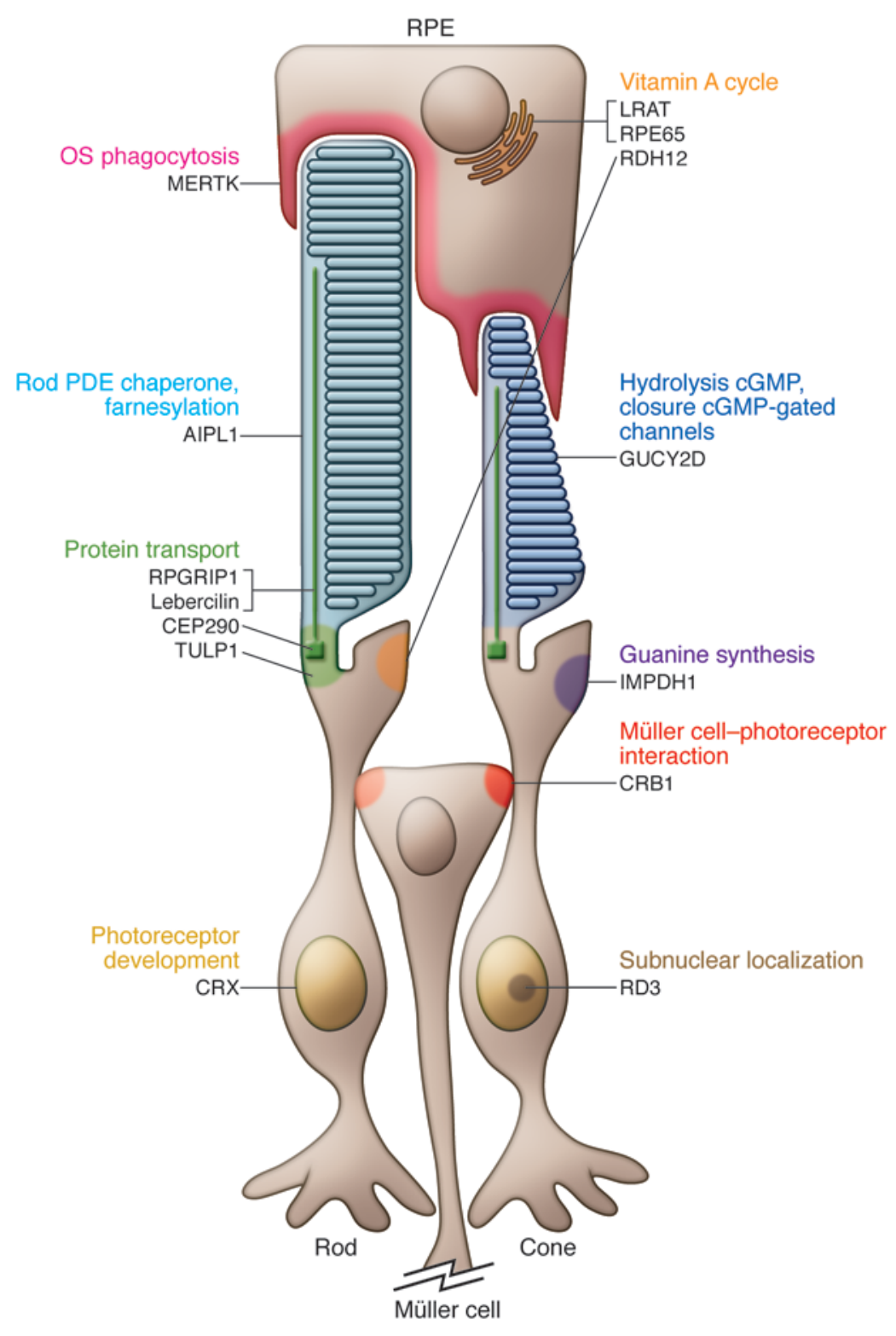

for approximately $70 \%$ of all LCA cases. The most frequently mutated genes are centrosomal protein $290 \mathrm{kDa}$ (CEP290; also known as NPHP6), guanylate cyclase $2 \mathrm{D}$ (GUCY2D), and crumbs homolog 1 (CRB1) (2). Mutations in the retinal pigment epithelium-specific protein $65 \mathrm{kDa}($ RPE65) gene account for $5 \%-10 \%$ of all LCA cases $(2,3)$. $R P E 65$ is highly expressed in the retinal pigment epithelium (RPE), where it encodes a retinoid isomerase enzyme essential for the production of chromophore, which forms the visual pigment in the rod and cone photoreceptors of the retina. Interestingly, the LCA-associated genes encode proteins with a wide variety of retinal functions, such as photoreceptor morphogenesis, phototransduction, vitamin A cycling, guanine synthe- sis, and outer segment (OS) phagocytosis (Figure 1). Recently, several mutations were identified that are likely to affect intraphotoreceptor ciliary transport processes.

Although nearly all identified LCA-associated genes are expressed preferentially or specifically in the retina, two genes recently identified as mutated in patients with LCA, CEP290 (4) and LCA5 (which encodes Lebercilin; ref. 5), are ubiquitously expressed. Furthermore, the identification of these genes as LCA associated highlights the important role of ciliary proteins in the pathogenesis of LCA, as has been observed for other retinal dystrophies (e.g., Bardet-Biedl and Usher syndromes). Mutation analysis in a French Canadian family identified an intronic mutation,

\section{Figure 1}

Spatial expression of the proteins encoded by LCA-associated genes. RPE65 and LRAT are located in the membranes of RPE cell endoplasmic reticulum. CEP290 is located in the basal bodies of rod and cone photoreceptors. Lebercilin, CEP290, and RPGRIP1 have prominent localization in the photoreceptor CC. GUCY2D localizes to the OS disc membranes of rod and cone photoreceptors. PDE, phosphodiesterase. Adapted with permission from Progress in Retinal and Eye Research (2).

c. $2991+1655 A>G$ (p.C998X), that results in the insertion of a cryptic exon in the CEP290 mRNA and introduces a premature stop codon in the protein. Strikingly, this mutation was found in $20 \%$ of LCA patients, rendering it one of the most important causes of LCA to date (4).

The diagnosis of LCA is based on clinical findings. Identification of the genetic cause by molecular genetic testing is important to both distinguish LCA from other retinal diseases and give a patient a more accurate visual prognosis based on genotype-phenotype correlations.

In this issue of the JCI, two independent research groups report significant new data on the molecular pathogenesis of LCA and on the potential of gene therapy in individuals with selected LCA-causing gene defects. Boldt et al. demonstrate a direct involvement of defective intraflagellar transport (IFT) in LCA and provide evidence that Lebercilin functions as an integral element of selective protein transport through photoreceptor cilia (6). Ashtari et al. demonstrate stable retinotopic functional improvement in humans after retinal gene therapy, as reflected by responses of the visual cortex (7).

\section{Some mechanisms implicated in the pathogenesis of LCA}

The mechanisms underlying LCA are multiple and not fully understood. Despite the striking genetic heterogeneity, the main clinical features of LCA can be categorized into a more limited number of phenotypes, a fact that points toward overlapping pathogenic disease mechanisms. Emerging data suggest that one of these possible mechanisms may affect transport across the photoreceptor connecting cilium (CC) - the sole link between the inner segment (IS) and OS of photoreceptors. As the photoreceptor IS (containing the metabolic machinery of the cell) and the OS (membranous disks containing phototransduc- 


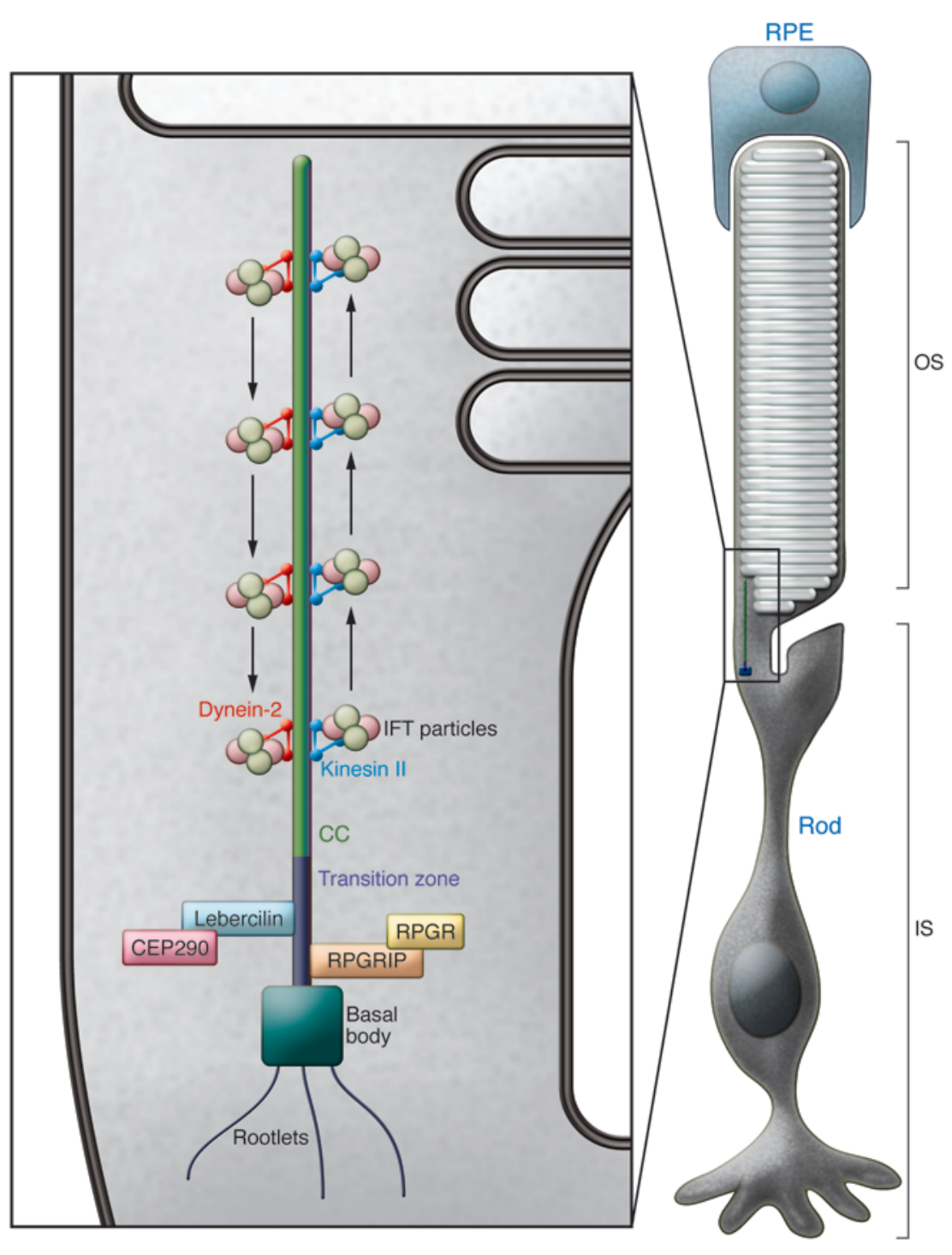

tion proteins) are linked solely by a CC, correct transport of proteins through the $\mathrm{CC}$ is crucial for the function and maintenance of the cell (Figure 2). Boldt et al. analyze how mutations in the LCA5 gene affect the connectivity of the encoded protein, Lebercilin, and provide evidence that Lebercilin functions as an integral element of selective protein transport through the photoreceptor CC (6). This study represents the first molecular confirmation that disrupted IFT - an elaborate mechanism that mediates the polarized trafficking of proteins required for efficient phototransduction - is directly implicated in LCA. The primary cilium of photoreceptors mediates polarized trafficking of proteins for efficient phototransduction. During ciliogenesis, protein complexes are transported distally for growth of the axoneme using IFT
(8). Since proteins and protein precursors are transported across the ciliary compartments via IFT, mutations in genes encoding proteins that participate in IFT can cause a spectrum of different ciliopathies.

Connecting Lebercilin to LCA. Lebercilin was first reported to localize to cilia of cultured cells and to the photoreceptor CC and was linked to ciliary and centrosomal function (9). It is noteworthy that dysfunction of primary cilia (an extension of the cell membrane formed by nucleation of microtubules) as a result of mutations in cilia-centrosomal proteins is associated with pleiotropic disorders. To understand the findings of Boldt et al. (6), it is important to remember that correct transport of proteins through the $\mathrm{CC}$ via the process of IFT is crucial for the function and maintenance of photoreceptors. Indeed, about

\section{Figure 2}

Photoreceptor CC. Cilia consist of a backbone (or axoneme), which contains microtubule doublets arranged in a circle. The cilium is anchored in the basal body, which regulates and organizes microtubule assembly. The photoreceptor CC is a specialized primary (nonmotile) cilium. It connects the photoreceptor IS, which contains the metabolic machinery of the cell, and the OS, which contains the photosensitive apparatus. As the OS is unable to synthesize proteins and lipids, the CC has an important role in transport from the IS to the OS. RPGR, retinitis pigmentosa GTPase regulator.

2,000 opsin molecules are transported per minute from the rod IS, where they are synthesized, via the CC, to the rod OS, where they maintain function and integrity. Therefore, any impairment of the IFT process leads to dysfunction of photoreceptors, ultimately leading to degeneration of the retina. For example, disruption of IFT in kinesin family member $3 a$ (Kif3a) conditional knockout mice (10) results in opsin accumulation in ISs and, consequently, photoreceptor degeneration. In retinitis pigmentosa GTPase regulator interacting protein 1 (Rpgrip1) knockout mice, the degeneration of photoreceptors - and, subsequently, all retinal layers - is rapid (11). Interestingly, a common allele of RPGRIP1L is a modifier of retinal degeneration severity in ciliopathies (12).

Boldt et al. report their use of a powerful combination of a quantitative affinity proteomics approach using $L C A 5$-encoded Lebercilin and two human LCA-causative mutations in LCA5, together with the development and detailed examination of a homozygous Lca5 gene trap mouse model (6). They convincingly demonstrated that Lebercilin physically interacts with the intracellular transport machinery. As LCAassociated LCA5 mutations abrogated this interaction, these results demonstrated a direct involvement of defective IFT in the LCA disease phenotype. Boldt et al. further demonstrated that physical association of Lebercilin with IFT proteins occurred in the retina. They assessed the effect of LCA-associated LCA5 mutations on the Lebercilin interactome and showed that mutations Lebercilin-p.P493TfsX1 and Lebercilin-p.Q279X caused the loss of all identified IFT and IFT-associated proteins from the protein complex. The observation that Lebercilin and IFT proteins partly colocalized in cilia of retinal cells indicates 
that Lebercilin and Lebercilin-associated proteins are likely to be essential for specialized transport by the IFT machinery. The authors also generated homozygous Lca5 gene trap mice, which exhibited phenotypes (restricted to the retina) characteristic of LCA in humans. The involvement of Lebercilin in the transport of phototransduction proteins along the CC was demonstrated by loss of transport of cone and rod opsins and reduced translocation of arrestin and transducin in response to light, thus pointing to a role for Lebercilin as a molecular scaffold, connecting the IFT core machinery to proteins involved in selecting and recruiting cargo.

Other CC-localized proteins connected to LCA. CEP290, RPGRIP1, and Tubby-like protein 1 (TULP1) are other recently identified LCA-associated proteins that localize to CC of photoreceptor cells. TULP1 is a member of the Tubby-like family of proteins that play roles in neuronal development and function. An important role for TULP1 in retinal differentiation (in line with the early onset of the retinal degeneration in LCA) has also been suggested (13). Absence of Tulp 1 in mice has been shown to induce mislocalization of rhodopsin to the plasma membrane of the ISs and budding into extracellular vesicles (14). CEP290 is a cilia-centrosomal protein that was initially identified as a tumor antigen. Mutations in CEP290, now known to be implicated in the pathogenesis of LCA, are also associated with syndromic ciliopathies (such as Joubert and Meckel-Gruber syndromes), as are mutations in the RPGRIP1-like (RPGRIP1L) gene. With the identification of mutations in LCA5, CEP290, RPGRIP1, and TULP1, the role of disrupted ciliary processes in the molecular pathogenesis of LCA is emphasized, pinpointing a growing group of LCA subtypes as ciliopathies.

\section{Advances in therapy for LCA}

Ocular gene therapy has been tried with success in mice, dogs, and, most recently, humans (15). It is most advanced for LCA caused by mutations in RPE65. Proof of principle for gene therapy came from studies in Briard dogs, a naturally occurring model of retinal degeneration resulting from mutations in RPE65 (16). Gene therapy using adeno-associated virus-mediated (AAVmediated) delivery of RPE65 was shown to restore visual function in Briard dogs (16), with sustained success over many years in 95\% of treated eyes (17). The results of three simultaneous phase I clinical treatment tri- als of AAV-mediated RPE65 gene therapy in humans $(18,19)$ demonstrated safety and showed slight improvement in vision in both bright and dim light. Clinical data on more than 30 patients are now available, showing stable clinical benefit in all patients, with no severe adverse effects $(20,21)$.

Ashtari et al. studied how the visual cortex responds to recovery of retinal function after prolonged sensory deprivation and provided the spectacular initial evidence that the visual cortex can be made responsive to visual input even after prolonged visual deprivation (up to 35 years; ref. 7). This study sheds light on the still-unanswered question concerning the Molyneux debate. More than 300 years ago, Molyneux formulated a problem as to "whether a man who has been born blind and who has learnt to distinguish and name a globe and a cube by touch, would be able to distinguish and name these objects simply by sight, once he had been enabled to see" (22). For modern neuroscientists, the answer to this question has an important bearing on contemporary issues concerning crossmodal identification and intermodal interactions - in other words, how the brain integrates information from the different senses. The results from the work of Ashtari et al. have a substantial effect on our understanding of the capacities of the visual cortex to be reactivated after visual deprivation. The study involved individuals with LCA2, a form of LCA caused by mutations in $R P E 65$, who were injected unilaterally with AAV carrying normal RPE65 cDNA (AAV2hRPE65v2) in their worst-seeing eye. Using psychophysical testing and functional MRI (fMRI) 1 year after the injection, Ashtari et al. provided an excellent demonstration that gene augmentation therapy in humans with LCA2 rendered both the retina and the visual cortex far more sensitive to dimmer light and lower-contrast stimuli (7). Cortical activation was found in regions corresponding to the area of the retina that had been exposed to AAV2-hRPE65v2, and the topography corresponded to visual field improvement. This is the first demonstration of stable, retinotopic improvement in humans for at least 2 years after retinal gene therapy, reflected by response of the visual cortex. The fMRI finding that the visual cortex can be reawakened after chronic visual deprivation in a rare eye disease may have much broader implications, providing promise for the outcome of gene therapy for both early- and late-onset retinal degenerative diseases.
Future directions. Except for gene therapy for LCA2, no substantial treatment or cure for LCA exists; thus, care is currently supportive. One of the key issues in the near future will be the extension of results gained in individuals with RPE65 mutations to those with mutations in other genes. Emerging data suggest that CEP290 may account for a substantial percentage of LCA cases (5). Clinical and laboratory studies suggest that patients with CEP290related LCA may be good candidates for gene therapy. Indeed, gene therapy of an RPE-expressed gene encoding a vitamin A cycle enzyme (RPE65), a photoreceptor gene encoding a phototransduction enzyme (GUCY2D; ref. 23), and a photoreceptor gene encoding a structural protein (RPGRIP1; ref. 11) have all been shown to be successful in different experimental models. These successes were reached in recessive disorders that were obviously more amenable to gene replacement strategies. However, large animal models are not available for GUCY2D and aryl hydrocarbon receptor interacting protein-like 1 (AIPL1) deficiency, and the canine model of RPGRIP1 deficiency has not yet been treated by gene therapy.

\section{Translating genotypic and phenotypic insights into major therapeutic advances}

Considering the clinical heterogeneity of LCA (24), a detailed genotype-phenotype correlation analysis is critical in order to understand the progression and pathogenesis of this devastating eye disease, as illustrated by the study by Boldt et al. on Lebercilin (6). Further elucidation of the molecular mechanisms of LCA in the retina and development of gene therapy approaches for different genetic subtypes of the disease are required. In particular, the status of remaining photoreceptors at the time of treatment will represent the most predictive parameter in terms of functional improvement. Moreover, the age of onset of profound visual impairment will determine the development of the visual cortex in the early years during the critical period. As LCA2 often spares significant visual function in the early years, sometimes into the second decade, this may represent a privileged situation for gene therapy: photoreceptors are affected secondary to RPE dysfunction, and cortical stimulation by formed images has occurred during the critical period, prior to the advent of legal blindness. The report by Ashtari et al. on 
cortical function restoration after therapy (7) brings great promise. Depending on the affected gene and severity of the phenotype, the extension of the landmark achievements in LCA2 to other types of LCA may prove more challenging depending upon the age of intervention.

\section{Acknowledgments}

The author thanks Katia Marazova (Institut de la Vision) for major help in preparing this commentary and Thierry Leveillard (Institut de la Vision) and Laurent Cohen (Institut du Cerveau et de la Moelle, Paris, France) for advice.

Address correspondence to: José-Alain Sahel, Institut de la Vision, Pierre and Marie Curie Medical School-Paris 6, 17 rue Moreau, 75012 Paris, France. Phone: 33153462504; Fax: 33140021499; E-mail: j-sahel@quinze-vingts.fr.

1. Stone EM. Leber congenital amaurosis-a model for efficient genetic testing of heterogeneous disorders: LXIV Edward Jackson Memorial Lecture, Am J Ophthalmol. 2007;144(6):791-811.

2. den Hollander AI, Roepman R, Koenekoop RK, Cremers FP. Leber congenital amaurosis: genes, proteins and disease mechanisms. Prog Retin Eye Res. 2008;27(4):391-419.

3. Stieger K, Lorenz B. Gene therapy for vision loss -- recent developments. Discov Med. 2010; 10(54):425-433

4. den Hollander AI, et al. Mutations in the CEP290 (NPHP6) gene are a frequent cause of Leber congenital amaurosis. Am J Hum Genet. 2006;79(3):556-561.

5 . Dharmaraj S, et al. A novel locus for Leber congenital amaurosis maps to chromosome 6q. Am J Hum Genet. 2000;66(1):319-326.

6. Boldt K, et al. Disruption of intraflagellar protein transport in photoreceptor cilia causes Leber congenital amaurosis in humans and mice.J Clin Invest. 2011;121(6):2169-2180.

7. Ashtari $\mathrm{M}$, et al. The human visual cortex responds to gene therapy-mediated recovery of retinal function. J Clin Invest. 2011;121(6):2160-2168.

8. Rosenbaum JL, Cole DG, Diener DR. Intraflagellar transport: the eyes have it. J Cell Biol. 1999; 144(3):385-388.

9. den Hollander AI, et al. Mutations in LCA5, encoding the ciliary protein lebercilin, cause Leber congenital amaurosis. Nat Genet. 2007;39(7):889-895.

10. Marszalek JR, et al. Genetic evidence for selective transport of opsin and arrestin by kinesinII in mammalian photoreceptors. Cell. 2000; 102(2):175-187.

11. Pawlyk BS. Replacement gene therapy with a human RPGRIP1 sequence slows photoreceptor degeneration in a murine model of leber congenital amaurosis. Hum Gene Ther. 2010;21(8):993-1004.

12. Khanna $\mathrm{H}$, et al. A common allele in RPGRIP1L is a modifier of retinal degeneration in ciliopathies. Nat Genet. 2009;41(6):739-745.

13. Milam AH, et al. Localization of tubby-like protein 1 in developing and adult human retinas. Invest Ophthalmol Vis Sci. 2000;41(8):2352-2356.

14. Hagstrom SA, Adamian M, Scimeca M, Pawlyk BS, Yue G, Li T. A role for the Tubby-like protein 1 in rhodopsin transport. Invest Ophthalmol Vis Sci. 2001; 42(9):1955-1962
15. Musarella MA, Macdonald IM. Current concepts in the treatment of retinitis pigmentosa.J Ophthalmol. 2011;2011:753547.

16. Acland GM, et al. Gene therapy restores vision in a canine model of childhood blindness. Nat Genet. 2001;28(1):92-95

17. Acland GM, et al. Long-term restoration of rod and cone vision by single dose $\mathrm{AAV}$-mediated gene transfer to the retina in a canine model of childhood blindness. Mol Ther. 2005;12(6):1072-1082.

18. Cideciyan AV. Human gene therapy for RPE65 isomerase deficiency activates the retinoid cycle of vision but with slow rod kinetics. Proc Natl Acad Sci U S A. 2008;105(39):15112-15117.

19. Hauswirth WW, et al. Treatment of leber congenital amaurosis due to RPE65 mutations by ocular subretinal injection of adeno-associated virus gene vector: short-term results of a phase I trial. Hum Gene Ther. 2008;19(10):979-990.

20. Cideciyan AV, et al. Vision 1 year after gene therapy for Leber's congenital amaurosis. $N$ Engl J Med. 2009;361(7):725-727.

21. Simonelli F, et al. Gene therapy for Leber's congenital amaurosis is safe and effective through 1.5 years after vector administration. Mol Ther. 2010; 18(3):643-650.

22. Stanford Encyclopedia of Philosophy. Molyneux's Problem. Web Site. http://plato.stanford.edu/ entries/molyneux-problem/. Updated July 1, 2010. Accessed April 14, 2011.

23. Williams ML, et al. Lentiviral expression of retinal guanylate cyclase-1 (RetGC1) restores vision in an avian model of childhood blindness. PLoS Med. 2006; 3(6):e201.

24. Hanein S, et al. Leber congenital amaurosis: comprehensive survey of the genetic heterogeneity, refinement of the clinical definition, and genotypephenotype correlations as a strategy for molecular diagnosis. Hum Mutat. 2004;23(4):306-317.

\title{
From probiotics to therapeutics: another step forward?
}

\author{
Fayez K. Ghishan'1 and Pawel R. Kiela ${ }^{1,2}$ \\ 1Department of Pediatrics, Steele Children's Research Center, and 2Department of Immunobiology, \\ University of Arizona Health Sciences Center, Tucson, Arizona, USA.
}

\begin{abstract}
Preclinical studies with probiotics continue to unravel mechanisms of cytoprotection and suggest that approaches utilizing microbial products as therapeutics in acute and chronic gastrointestinal disorders could be effective. However, clinical trials using these bacteria have thus far been inconsistent. In this issue of the JCI, Yan et al. describe a novel mechanism of cytoprotection by $\mathrm{p} 40$, a soluble product of Lactobacillus rhamnosus GG, mediated via EGFR. The efficacy of $\mathrm{p} 40$ in three models of chemically induced colitis indicates tremendous therapeutic potential, though this finding will need to be verified in human patients.
\end{abstract}

The human body as a superorganism A new view of human body has emerged as a result of the work of the NIH Human

Conflict of interest: The authors have declared that no conflict of interest exists.

Citation for this article: J Clin Invest. 2011; 121(6):2149-2152. doi:10.1172/JCI58025
Microbiome Project (HMP), the European Metagenomics of the Human Intestinal Tract (MetaHIT) project, the International Human Microbiome Consortium (IHMC), and individual research laboratories. It is a picture of a superorganism in which a large number of different organisms coexist as one. In this superorganism, only $10 \%$ of cells represent Homo sapiens (1), with millions of microbial genes outnumbering the "mere" 20-25 thousand genes that constitute the human genome (2). The vast majority of the microorganisms coinhabiting humans are located within the confines of the gastrointestinal tract. Termed the gut microbiota, they have a collective mass of approximately $1.5 \mathrm{~kg}$, and together with the gastrointestinal tract itself they create the most metabolically active system within human body. The gut microbiota is a very diverse, complex, and dynamic system forming varying niches along the GI tract segments, and its makeup and function are affected by host genetics, environment, diet, and disease states. Humans have coevolved with their microbial component over two 